\title{
High incidence of preterm births and early losses in pregnancy after in vitro fertilisation
}

\author{
AUSTRALIAN IN VITRO FERTILISATION COLLABORATIVE GROUP
}

\begin{abstract}
A fertilisation cohort of 244 pregnancies resulting from in vitro fertilisation was reported to a national register by eight units specialising in in vitro fertilisation. Early pregnancy losses were high, with $5 \%$ tubal ectopic pregnancies, $18 \%$ biochemical pregnancies, and an incidence of spontaneous abortion of $27 \%$. Among pregnancies of at least 20 weeks' gestation $22 \%$ were multiple, with 26 pairs of twins and four sets of triplets. The incidence of preterm births was more than three times higher than in the general population. Low birthweight rates were also higher, owing both to preterm births and to multiple pregnancies. The sex ratio and the incidence of major congenital malformations were similar to those in naturally conceived pregnancies.

In this series the high fetal losses at all stages of pregnancy suggested maternal reproductive causes and should not be interpreted as being due to factors peculiar to in vitro fertilisation. Further analysis will be necessary when larger numbers are available.
\end{abstract}

\section{Introduction}

At this relatively early stage in in vitro fertilisation most studies have concentrated on the methods of achieving pregnancies. Very few reports ${ }^{12}$ have examined the outcome of these pregnancies beyond providing summary figures, partly because the numbers in individual in vitro fertilisation units are still quite small.

To enhance the likelihood of detecting any increases in adverse outcomes, in vitro fertilisation units in Australia (through the Fertility Society of Australia) agreed to pool their data and requested that the National Perinatal Statistics Unit should collaborate with them in developing a voluntary, national register of pregnancies resulting from in vitro fertilisation. The main objective of the register was to discover whether the incidence of congenital malformations was increased, but other outcomes of pregnancy were also recorded. We report the findings in the first group of pregnancies notified to the register.

\section{Subjects and methods}

The first pregnancy achieved by in vitro fertilisation in Australia occurred in 1979. Eight units with pregnancies completed before the

\footnotetext{
Members of the collaborative group were: Dr P A L Lancaster (National Perinatal Statistics Unit, University of Sydney); Dr W I H Johnston (Reproductive Biology Unit, Royal Women's Hospital, Melbourne); Professor C Wood (Queen Victoria Medical Centre, Melbourne); Associate Professor D M Saunders (Royal North Shore Hospital, Sydney); Professor $W R$ Jones (Flinders Medical Centre, Adelaide); Dr J F Kerin (Queen Elizabeth Hospital, Adelaide); Dr J L Yovich (PIVET Laboratory and University of Western Australia); Dr J F Hennessey (Queensland Fertility Group, Brisbane); Professor J F Correy (Queen Alexandra Hospital, Hobart).
}

This report was prepared by Dr Lancaster.

Correspondence and requests for reprints to: Dr P A L Lancaster, National Perinatal Statistics Unit, School of Public Health and Tropical Medicine, University of Sydney, 2006, NSW, Australia. end of 1983 contributed data to the register. The laboratory and clinical aspects of in vitro fertilisation in one of these units have been re- $\frac{7}{0}$ viewed elsewhere. ${ }^{3}$ Varying criteria are used by the units to select $\frac{C}{}$ infertile couples for attempts at in vitro fertilisation. As a result $\overline{\bar{N}}$ outcomes of pregnancy in each unit may be influenced by the relative $\frac{\rho}{\sigma}$ distribution of the various causes of infertility. The main causes $\stackrel{\odot}{\mathscr{D}}$ were tubal abnormalities due to pelvic inflammatory disease, previous $\cong$ ectopic pregnancy, or sterilisation. Abnormalities of sperm and endometriosis occurred less commonly, while in some couples there $\vec{O}$ were multiple causes or the cause of infertility was unknown.

Data items on forms used to notify pregnancies include: parentale ages and the number of previous pregnancies; the causes and duration of infertility; the drugs used to induce ovulation, the laparoscopy 3 . cycle in which pregnancy occurred, and the number of oocytes collected; the use of donor sperm, oocytes, and embryos and whether embryos had been frozen; the number of embryos replaced in the uterus after in vitro fertilisation; the date of fertilisation; any drugs $O$ used during the luteal phase; and the number of gestational sacs seen $\omega$ at the initial ultrasound examination. The outcomes recorded are: $\vec{\overrightarrow{ }}$ biochemical pregnancies, ectopic pregnancies, spontaneous abortions, stillbirths and live births, multiple pregnancies, and the infants' date of $\mathrm{O}$ birth, sex, birth weight, and method of delivery, as well as the presence $\supset$ of any congenital malformations. No identifying data about families $\widetilde{\sim}$ are held in the register, but an in vitro fertilisation unit number for $O$ each couple allows exchange of information between units and the National Perinatal Statistics Unit. Forms are filled in by staff in the in vitro fertilisation units, using their records and follow up information from referring doctors. The completeness and accuracy of data $\vec{\bullet}$ are maintained by requesting additional information if data are missing $\infty$ or ambiguous and by returning computer printouts to each unit for. checking. Any corrections are then sent to the National Perinatal Statistics Unit. Data on survival at 1 year of age and the diagnosis of any congenital malformations beyond the early neonatal period will, in due course, be sought from referring doctors.

The following definitions are used for outcomes of pregnancy.

Biochemical pregnancy is recorded when the evidence of pregnancy is derived only from serum $\beta$ human chorionic gonadotrophin concen- $\overrightarrow{\vec{\theta}}$ trations, measured no earlier than day 16 (after ovum pick up) of the 3 luteal phase and in the absence of chorionic villi should curettage be performed. Concentrations should be greater than the value which is two standard deviations below the mean for the laboratory assay in the absence of injections of chorionic gonadotrophin during the luteal phase. Using this definition, $\beta$ human chorionic gonadotrophin concentrations in non-pregnant women are lower than two standard 0 deviations below the mean.

Ectopic pregnancy is defined as pregnancy occurring outside the uterus.

Spontaneous abortion is recorded for fetal loss at less than 20 weeks $\mathrm{O}$ of gestation (measured from the first day of the last menstrual period) in a pregnancy detected clinically or by ultrasound.

Stillbirth is defined as fetal death in a pregnancy of at least 20 weeks $D$ of gestation.

Live birth is defined as an infant with signs of life after a pregnancy of at least 20 weeks of gestation.

Preterm birth is defined as a live birth or stillbirth of less than $37 \mathrm{~N}$ completed weeks of gestation.

Oocyte recovery and fer+ilisation in in vitro fertilisation usually occur on day 14 of the menstrual cycle. So that the duration of preg- $\frac{}{\Phi}$ nancies achieved by the technique could more readily be compared $\stackrel{\oplus}{?}$ with other pregnancies, the gestational age was derived by adding 14 days to the interval between the dates of fertilisation and birth. Possibly the duration of pregnancy in some spontaneous abortions $\overrightarrow{\mathbb{D}}$ reported by referring doctors was already based on gestational age $\stackrel{?}{\mathbb{P}}$ rather than fertilisation age, thereby overestimating the calculated $\varrho$ gestational age by two weeks.

Early pregnancy losses were over-represented in the total figures for pregnancies completed during 1980-3 because some pregnancies resulting from fertilisation in 1983 were not completed by the end of that year. Analysis of outcomes related to gestational age was therefore

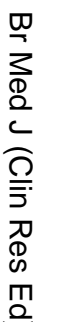


BRITISH MEDICAL JOURNAL VOLUME 291 26 OCTOBER 1985

restricted to a cohort of pregnancies in which fertilisation occurred before 1 April 1983. All these pregnancies were completed before the end of 1983

\section{Results}

There were 244 pregnancies in the fertilisation cohort, which were among a group of 322 pregnancies reported as completed during 1980-3.

\section{EARLY PREGNANCY LOSSES}

The incidence of the various outcomes of pregnancy could be expressed in several ways, depending on which denominator was used. Overall, of the 244 pregnancies (table I) $135(55 \%$ ) resulted in live births, $43(18 \%)$ were biochemical pregnancies, and $13(5 \%)$ were ectopic pregnancies. The figures for biochemical pregnancies in natural conceptions are not known reliably, so they should be excluded from

TABLE I-Outcome of pregnancies resulting from in vitro fertilisation in fertilisation cohor

\begin{tabular}{lrrr}
\hline Outcome & No & $\%$ & $\%^{*}$ \\
\hline Biochemical pregnancy & 43 & 18 & \\
Ectopic pregnancy & 13 & 5 & \\
Spontaneous abortion & 50 & 21 & 27 \\
Stillbirth & 3 & 1 & 2 \\
Live birth & 135 & 55 & 72 \\
\hline Total & 244 & 100 & 100 \\
\hline
\end{tabular}

*Excluding biochemical and ectopic pregnancies from denominator.

Note: Pregnancies resulted in total of 172 live and stillborn infants.

calculations of spontaneous abortion rates when comparing in vitro fertilisation and natural pregnancies. Ectopic pregnancies should also be excluded from such comparisons because they are not at risk for spontaneous abortion. These exclusions left 188 pregnancies as the denominator for calculating the incidence of spontaneous abortion, which was $27 \%$. These results indicate that both ectopic pregnancies and spontaneous abortions were more common in pregnancies resulting from in vitro fertilisation than in published figures for natural pregnancies. ${ }^{4}$ In pregnancies of at least 20 weeks' gestation stillbirths occurred in three out of 172 total births, a rate of 17 per 1000 total births. Again, these fetal losses were about twice as great as those occurring in the general population. ${ }^{5}$

Table II shows a trend of increasing incidence of spontaneous abortions in older women, similar to the findings in natural pregnancies.

TABLE II-Maternal age and outcome of pregnancy (excluding biochemical pregnancies). Results expressed as number ( $\%$ ) of pregnancies*

\begin{tabular}{lllll}
\hline & \multicolumn{4}{c}{ Maternal age (years) } \\
\cline { 2 - 5 } Outcome & $<25$ & $25-29$ & $30-34$ & $35-39$ \\
\hline Ectopic pregnancy & 1 & 2 & 5 & 5 \\
Spontaneous abortion & $1(20)$ & $13(24)$ & $26(28)$ & $10(29)$ \\
Pregnancy $\geqslant 20$ weeks & $4(80)$ & $42(76)$ & $68 \dagger(72)$ & $24(71)$ \\
\hline Total & $6(100)$ & $57(100)$ & $99(100)$ & $39(100)$
\end{tabular}

*Ectopic pregnancies excluded from calculations of percentages.

†Includes three pregnancies resulting in at least one stillbirth.

\section{MULTIPLE BIRTHS}

Multiple births occurred in $30(22 \%)$ of the 138 pregnancies of at least 20 weeks' gestation, twins in 26 cases (19\%), and triplets in four $(3 \%)$. Not all early pregnancies were screened by ultrasound, so undetected multiple pregnancies may have been included among the early fetal losses. This high incidence of multiple pregnancy (compared with about $1 \%$ in the Australian population) was consistent with usually transferring more than one embryo after fertilisation. Of the 30 pregnancies fertilised in 1979-81 and reaching 20 weeks' gestation, $3(10 \%)$ were multiple; while of 108 fertilised in 1982 and early 1983, 27 $(25 \%)$ were multiple.

One twin pregnancy occurred after transfer of a single embryo, and another monozygotic pregnancy occurred after transfer of two embryos. ${ }^{6}$ Zygosity was not studied routinely in twins of the same sex.

\section{PRETERM BIRTHS}

Multiple pregnancy was a factor in the higher incidence of preterm births. In twin pregnancies six of the deliveries $(23 \%)$ were preterm, as were all four triplet pregnancies (table III). In single pregnancies $20(19 \%)$ of the infants were delivered before term-more than three times higher than the comparable figure $(6.2 \%)$ in an Australian population. ${ }^{7}$ While most $(15 / 20)$ of these single preterm births occurred at 32-36 weeks of gestation, $5 \%$ of all single births occurred before 32 weeks.

TABLE III-Durations of single and multiple pregnancies of at least 20 weeks' gestation resulting from in vitro fertilisation. Results expressed as number (\%) of pregnancies

\begin{tabular}{lcccc}
\hline $\begin{array}{l}\text { Gestational age } \\
\text { (weeks) }\end{array}$ & Total & Single & Twin & Triplet \\
\hline$<32$ & $9(7)$ & $5(5)$ & $2(8)$ & $2(50)$ \\
$32-36$ & $21(15)$ & $15(14)$ & $4(15)$ & $2(50)$ \\
$\geqslant 37$ & $108(78)$ & $88(81)$ & $20(77)$ & \\
\hline Total & $138(100)$ & $108(100)$ & $26(100)$ & $4(100)$ \\
\hline
\end{tabular}

\section{BIRTH WEIGHT}

The high incidence of preterm births was also reflected in the birth weight of the infants. Among live born and stillborn infants with recorded birth weights the overall incidence of low birth weight (less than $2500 \mathrm{~g}$ ) was $30 \%(50 / 167)$. In singletons it was $19 \%(20 / 106)$, in twins $42 \%(21 / 50)$, and in triplets $82 \%(9 / 11)$ (table IV). Obvious fetal growth retardation (less than the 10 th percentile) was not evident, although the number of infants was relatively small.

TABLE IV-Birth weights of infants in single and multiple pregnancies resulting from in vitro fertilisation. Results expressed as number (\%) of infants

\begin{tabular}{lcccc}
\hline Birth weight $(\mathrm{g})$ & Total & Single & Twin & Triplet \\
\hline $500-999$ & $3(2)$ & $3(3)$ & & \\
$1000-1499$ & $10(6)$ & $2(2)$ & $4(8)$ & $4(36)$ \\
$1500-1999$ & $10(6)$ & $3(3)$ & $2(4)$ & $5(46)$ \\
$2000-2499$ & $27(16)$ & $12(11)$ & $15(30)$ & $2(18)$ \\
$\geqslant 2500$ & $117(70)$ & $86(81)$ & $29(58)$ & $11(100)$ \\
\hline Total & $167(100)$ & $106(100)$ & $50(100)$ & 1 \\
\hline Not stated & 5 & 2 & 2 & 1
\end{tabular}

SEX

The 186 infants born during 1980-3 comprised almost equal numbers of boys (92) and girls (94). Among single births 55 were boys and 59 girls, while for twins and triplets the figures were 30:24 and 7:11 respectively. Of the 27 sets of twins, both twins were boys in 10, both were girls in eight, and nine pairs had both a boy and a girl. There were six sets of triplets: three sets consisted of two boys and a girl, one set a boy and two girls, and the other two sets were all girls.

\section{CONGENITAL MALFORMATIONS}

Major congenital malformations were reported in two of the 186 infants born during 1980-3, an incidence of $1 \cdot 1 \%$. Comparable incidence rates in Australia are $1 \cdot 5-2 \cdot 0 \%$ (National Perinatal Statistics Unit, unpublished data). A dizygotic twin girl had transposition of the great vessels, and another girl had congenital dislocation of the hip. While amniocentesis was carried out in some pregnancies, early pregnancy losses were not systematically examined for chromosomal abnormalities. 


\section{METHOD OF DELIVERY}

Of 136 pregnancies in which the method of delivery was recorded, caesarean section was performed in $45(33 \%)$ and vaginal delivery in $91(67 \%)$.

\section{Discussion}

Women who become pregnant after in vitro fertilisation are usually older than their peers who conceive naturally, have atypical reproductive histories, and have been managed by novel techniques. Likewise, their fetuses have initially developed and been nurtured in potentially hazardous conditions, being exposed to various physical manipulations, to a possibly inadequately prepared uterine environment, and often to various hormonal and drug regimens. Hence it might be expected that outcomes of pregnancy resulting from in vitro fertilisation would differ from those after natural conceptions, especially when the initial experiences of in vitro fertilisation units are being examined.

These factors must be considered when interpreting these results. Pregnancy would not usually occur without in vitro fertilisation, so it is difficult to obtain a suitable group for comparison of outcomes. Comparison with the general obstetric population is not ideal, but it does at least put the overall results of in vitro fertilisation in perspective, even if the reasons for various outcomes of pregnancy cannot readily be determined. Future studies of larger numbers of pregnancies will need to address these problems.

While the number of pregnancies resulting from in vitro fertilisation is still relatively small, these Australian data show an emerging pattern of high pregnancy losses at all stages. The incidence of ectopic pregnancies was much higher than usual; spontaneous abortions were about twice as frequent as in natural pregnancies; stillbirths, although few in number, appeared to be more common; and, perhaps most surprisingly, the incidence of preterm births in single pregnancies was more than three times that in comparable population based figures. Also, given a high incidence of preterm, low birthweight infants, neonatal and thus perinatal mortality will be increased, but information on survival of liveborn infants was not available for this analysis.

Biochemical pregnancies after in vitro fertilisation cannot readily be compared with natural pregnancies, but more detailed analysis may shed light on the factors contributing to losses in the first few weeks after conception. Differing protocols for hormonal assessments in each in vitro fertilisation unit are likely to influence the detection of biochemical pregnancies.

The high incidence of tubal ectopic pregnancy after in vitro fertilisation may be related either to the techniques of embryo transfer or to underlying tubal disease and previous tubal surgery. The position of the catheter when the embryo is transferred into the uterus seems to be important. Radio-opaque dye has been shown to enter the oviduct when the embryo is replaced. ${ }^{8}$ The volume of medium and the rate at which it is injected are other factors that should be considered. In addition, the endosalpinx was frequently overstimulated by hormones given to the women during the treatment cycle, which may thus provide an inappropriate implantation site.

Reasons for the apparently higher incidence of spontaneous abortion in these pregnancies are more difficult to determine. Ascertainment of spontaneous abortions-even if based on clinical and ultrasound criteria-will probably be improved when pregnancies are followed up prospectively from conception. Just as regular hormonal monitoring in early pregnancy improved detection of biochemical pregnancies before the onset of the usual clinical features, so also may early spontaneous abortions be better recognised. Some causes of infertility may be associated with a greater risk of abortion. It is also possible that the greatly increased occurrence of multiple pregnancies after in vitro fertilisation may be a factor. Because ultrasound is frequently used early in pregnancies achieved by in vitro fertilisation to assess the appearance and number of gestational sacs, further analysis of these pregnancies should improve understand- ing of how often early abortion occurs in multiple pregnancies.

Maternal age is an important confounding variable influencing analysis of spontaneous abortion. Despite the older ages of women with pregnancies resulting from in vitro fertilisation the $c$ incidence of spontaneous abortion still seems high. These abortions occurred at all gestational ages and merged with the more $\overline{\bar{J}}$ advanced gestational ages of the preterm births, suggesting that underlying maternal reproductive characteristics were probably $\mathbb{E}$ more important than factors peculiar to in vitro fertilisation. Nevertheless, without systematic study of abortuses in these pregnancies it would be premature to exclude an increased occurrence of chromosomal abnormalities. As yet, however, there is no indication of any increased incidence of such abnormalities in pregnancies progressing beyond 20 weeks of gestation.

Preterm births were also distributed across a broad range of gestational ages from 20 to 36 weeks. The incidence of preterm birth was only slightly higher in twin than in single pregnancies. Some surviving single fetuses may originally have been multiple pregnancies in which one or more gestational sacs had been resorbed. ${ }^{9}$ If so the preliminary results do not suggest that fetal growth was adversely affected. Information about elective induction of labour was not collected, but it would hardly be surprising if this occurred more often after in vitro fertilisation, particularly among the earlier confinements. Maternal stress may also be a contributing factor. The high caesarean section rate after in vitro fertilisation may reflect both obstetric complications and more frequent intervention in high risk pregnancies.

The pronounced increase in multiple pregnancies was directly attributable to frequently replacing more than one embryo after fertilisation. ${ }^{10}$ One monozygotic twin pregnancy, however, was recorded after replacement of a single embryo. The ratio of $N$ twins of like to unlike sex was $2: 1$, whereas equal numbers would be expected if all were dizygotic twins resulting from fertilisation of more than one oocyte. Thus other monozygotic twins may have occurred. Early anecdotal and published ${ }^{111}$ reports showed an excess of girls in pregnancies resulting from in vitro fertilisation. With the larger numbers reported here the sex ratio was similar to that found in natural pregnancies.

There are several possible reasons why an increased incidence of chromosomal abnormalities or structural congenital malformations might occur after in vitro fertilisation. Both maternal and paternal ages are generally more advanced, increasing the risk of some chromosomal abnormalities and mutational events. Multiple pregnancies are associated with a higher incidence of congenital malformations. ${ }^{12}$ The laboratory conditions and procedures, including polyspermy, ${ }^{13}$ are potential causes, but embryos recognised as abnormal would not be transferred into the uterus. Some of the drugs used in the management of early pregnancy ${ }^{14}$ may cause masculinisation of fetuses. Against this background it is reassuring that the incidence of such abnormalities and malformations was no higher after in vitro fertilisation than in other pregnancies, but numbers were small and, as mentioned above, cytogenetic studies were not routinely performed on abortuses or other products of conception.

The logistic problems in developing a national register of $\mathrm{N}$ pregnancies resulting from in vitro fertilisation are considerable, $D$ particularly as many women successfully treated in in vitro fertilisation units are subsequently confined at other hospitals, necessitating requests for details of outcome. Despite these problems the advantages of combining the experience of all in vitro fertilisation units to evaluate a new therapeutic approach in managing infertile couples appear to justify the effort. This early analysis has yielded both expected and unexpected results. Further analy- $\mathbb{D}$ ses are needed with larger numbers of pregnancies, and it will: be interesting to see whether similar studies from other centres confirm our initial findings. It may be that some avoidable factors influencing the high incidence of preterm birth can be determined.

As well as its value for studying the factors associated with ${ }^{0}$ various outcomes of pregnancy, the register has the potential o benefit of indirectly identifying cases for follow up studies, which would then be conducted through the in vitro fertilisation

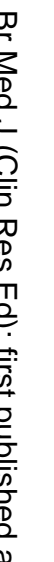


units and referring doctors. Any such studies of developmental outcomes will be carried out by the in vitro fertilisation units or groups working with them. The register would facilitate rapid assessment of numbers and where the families were living. Viable national or regional registers would be a prerequisite for international collaborative studies of pregnancies achieved by in vitro fertilisation.

We thank members of the in vitro fertilisation units for providing data to the register, and staff of the National Perinatal Statistics Unit for data processing and other work. The National Perinatal Statistics Unit is funded by a grant from the Commonwealth Department of Health.

\section{References}

1 Wood C, Trounson A, Leeton JF, et al. Clinical features of eight pregnancies resulting from in vitro fertilization and embryo transfer. Fertil Steril 1982;38: 22-9.
2 Edwards RG, Steptoe PC. Current status of in-vitro fertilisation and implantation of human embryos. Lancet 1983;ii:1265-9.

Lopata A. Concets in human in vitro fertilization and embryo transfer. Fertil Steril 1983;40:289-301.

Jansen RPS. Spontaneous abortion incidence in the treatment of infertility. $\mathrm{Am} \mathfrak{3}$ Obstet Gynecol 1982;143:451-73

Australian Bureau of Statistics. Perinatal deaths, Australia, 1982. Canberra: Commonwealth Government Printer, 1984

6 Yovich JL, Stanger JD, Grauaug A, et al. Monozygotic twins from in vitro fertilization. Fertil Steril 1984;41:833-7.

ealth Commission of Victoria. Survey of perinatal deaths in Victoria. Melbourne:

8 Steptoe PC. Discussion on the laparoscopic recovery of oocytes. In: Edwards RG, Purdy JM, eds. Human conception in vitro. London: Academic Press, 1982: 119-32.

Kerin JF, Warnes GM, Quinn PJ, et al. Incidence of multiple pregnancy after in-vitro fertilisation and embryo transfer. Lancet 1983;ii:537-40.

10 Craft I, Porter R, Green S, et al. Success of fertility, embryo number, and invitro fertilisation. Lancet $1984 ; \mathrm{i}: 732$.

11 Speirs AL, Trounson A, Warnes GM, Yovich JL, Saunders DM, Chen C. Summary of results. In: Wood C, Trounson A, eds. Clinical in vitro fertilization.

12 Warkany J. Congenital malformations. Chicago: Year Book Medical Publishers,

13 Wood C. The Tracy Maund obstetrical lecture. The beginning of life. Med f Aust $1981 ; \mathrm{i}: 305-12$.

14 Yovich J, Puzey A, De'Atta R, Roberts R, Reid S, Grauaug A. In-vitro fertilisation pregnancy with early progestagen support. Lancet 1982 ;ii:378-9.

(Accepted 1 August 1985)

\begin{abstract}
Decisions about coronary care made by 39 randomly selected general practitioners in New Zealand over one year were investigated. Demographic variables and variables relating to the patient's condition, history, and social circumstances were assessed for 113 patients, and practitioners' belief about the relevance of each variable to the decision was elicited. A comparison was made between the objective criteria found to be related to the decision, the criteria believed by the practitioners to be relevant to the decision, and a theoretical model of what should govern the decision to admit. The theoretical model was found to be built into the beliefs of the practitioners, but the objective model was far simpler.

Further research is needed to determine whether the use of the more complex, theoretical models will confer any benefit, in terms of patient wellbeing, over the pragmatic model in use at present.
\end{abstract}

\section{Introduction}

In recent years there has been considerable discussion about decision making by doctors. ${ }^{1-5}$ Most of this discussion, however,

Christchurch Clinical School, Christchurch, New Zealand

A E STEPHENSON, MB, DIPOBST, department of community health

D M FERGUSSON, BA, department of paediatrics

A R HORNBLOW, PHD, associate professor, department of community health

$D W$ BEAVEN, FRCP, FRACP, department of medicine

$S$ J CHETWYND, PHD, department of community health

Correspondence and requests for reprints to: Associate Professor A R Hornblow, Department of Community Health, PO Box 4345, Christchurch, New Zealand. has focused on the theoretical criteria that ought to govern decision making rather than on how doctors actually arrive at decisions. In general, three decision models can be identified that may be applied to any clinical decision. The first is the theoretical and prescriptive model proposed by accepted medical opinion; the second is based on the beliefs of individual general practitioners about the criteria that are relevant to their decision making; and the third is the decision model that may be derived from statistical analysis of the factors associated with various decisions. Clearly, considerable discrepancies may occur between these three decision models as the beliefs of individual general practitioners may not exactly reflect current medical opinion and the actual decisions made may differ from the beliefs held by the individual general practitioner or those expressed by accepted authorities.

We report a study of decisions on coronary care made by a sample of general practitioners in New Zealand. We set out to examine three questions. Firstly, what factors discriminate patients suspected of having acute myocardial infarction who are admitted to hospital from those who are treated at home? Secondly, what factors do general practitioners believe are relevant in the decision to admit patients with acute myocardial infarction to hospital or to treat them at home? Thirdly, how well do the objective factors that actually discriminate between those patients treated in hospital and those treated at home compare with the beliefs of general practitioners and the accepted medical opinion about the criteria that should be used in making the decision to admit the patient to hospital ?

\section{Method}

SAMPLE

We studied 113 consecutive patients presenting with suspected acute myocardial infarction. These patients represented all such 